\title{
DISEÑO ESTADÍSTICO APLICADO A LAS PROPIEDADES HÍDRICAS COMO CONTROL DE TRATAMIENTOS DE CONSOLIDACIÓN Y/O HIDROFUGACIÓN SOBRE GRANITOS
}

\author{
STATISTICAL DESIGN APPLIED TO HYDRIC PROPERTY BEHAVIOUR \\ FOR MONITORING GRANITE CONSOLIDATION AND/OR WATER-REPELLENCY \\ TREATMENTS
}

\author{
A. C. ÍNIIGO(*) ${ }^{(* *)}$, S. VICENTE-TAVERA(*)(**), V. RIVES ${ }^{(*)}(* * *)$
}

Recepción/Received: 16-II-05

Aceptación/Accepted: 30-VIII-05

\section{RESUMEN}

La adecuación de tratamientos de conservación (consolidación y/o hidrofugación), realizados sobre granitos ampliamente utilizados en el patrimonio cultural, se evaluó por los cambios experimentados en las características intrínsecas de estas rocas (capacidad de imbibición, porosidad abierta, porosidad total accesible al agua, permeabilidad al vapor de agua y coeficiente de absorción capilar). Se realizó un análisis estadístico de los valores obtenidos de estas propiedades, que muestra diferentes capacidades para el transporte de fluidos a través de las rocas, indicando que el tratamiento con consolidantes y posterior aplicación de hidrofugantes tiene mayor efecto que los dos aplicados independientemente y mejora notablemente sus propiedades como material de construcción.

Palabras clave: rocas ornamentales, granitos, tratamientos de consolidación e hidrofugación, porosidad.

\section{SUMMARY}

The suitability of granite conservation treatments (consolidation and/or water-repellency) widely used to protect heritage structures was evaluated on the basis of the changes occurring in the intrinsic characteristics of the stone (imbibition capacity, open porosity, total water-accessible porosity, water vapour permeability and capillar absorption coefficient). A statistical analysis conducted of the values obtained for these properties, which showed differences in fluid flow through the rocks, found that the application of consolidants followed by water repellency treatment was more effective than either procedure alone, substantially enhancing the properties of the stone from the standpoint of its use as a construction material.

Keywords: ornamental rocks, granites, consolidation and waterrepellency treatments, porosity.

\section{INTRODUCCIÓN}

Los consolidantes penetran a través de la red poral directamente conectada a la superficie de una roca y actúan como cemento artificial. Su penetración no es un proceso homogéneo y va a depender de la composición y morfología del producto una vez solidificado, así como del tamaño y grado de conexión de los poros de la roca tratada; es decir, la eficacia de estos tratamientos no depende exclusivamente de su composición química, sino también de sus propiedades texturales y mineralógicas de las rocas (1).

\section{INTRODUCTION}

Consolidants penetrate directly into the network grid accessible from the surface of a rock, acting like an artificial cement. Such penetration is not a homogeneous process, varying rather with the composition and morphology of the product after curing, and with pore size and connectivity in the rock treated; in other words, the effectiveness of such treatments does not depend exclusively on their chemical composition, but also on the texture and mineralogical properties of the stone (1).

\footnotetext{
(*) Unidad Asociada IRNA-CSIC Salamanca/Universidad de Salamanca (España),

(**) Instituto de Recursos Naturales y Agrobiología (CSIC). Salamanca (España).

${ }^{(* * *)}$ Deparțamento de Estadística, Universidad de Salamanca (España).

${ }^{(* * *)}$ Departamento de Química Inorgánica, Universidad de Salamanca (España).

Persona de contacto/Corresponding author: adolfo@usal.es (A. C. İñigo).
} 
Las propiedades hídricas determinadas antes y después de la aplicación de los tratamientos han sido determinadas por algunos autores (1-8) para evaluar la eficacia de los tratamientos de consolidación y/o hidrofugación. Martín y col. (2) han diseñado un instrumento para la medida de la determinación de la absorción de agua por capilaridad y por inmersión total, la sorción y desorción de agua y la permeabilidad al vapor de agua, que presenta un control automático por medio de ordenador y permite termostatizarlo en un amplio intervalo de temperaturas.

Por otra parte, en la aplicación tanto de consolidantes silico-orgánicos como hidrofugantes de naturaleza polisiloxánica, la permeabilidad al vapor de agua controla la transpiración natural de una piedra. Si la permeabilidad es extremadamente baja, la circulación de líquidos estará dificultada, sin embargo, gases y vapores serán capaces de circular libremente. El uso de hidrofugantes tiene este objetivo, evitar la penetración de líquidos, permitiendo la eliminación de gases, evitando así la tensión producida por aumento de volumen de los mismos a causa de una subida de temperatura (9).

La porosidad total accesible en agua de un material es el resultado de la suma de la porosidad "atrapada" (no conectada a la superficie externa y difícilmente accesible a los fluidos) más la porosidad abierta (conectada a la superficie externa y de fácil acceso a los fluidos). Así, la porosidad abierta controla el transporte de fluidos a través de la piedra a temperatura y presión ambientales $(9,10)$.

En el presente trabajo se estudia el efecto de los tratamientos de consolidación y/o hidrofugación sobre 5 muestras de facies graníticas "frescas", previamente envejecidas o no en una cámara climática, bajo condiciones controladas, mediante un análisis cuidadoso de los cambios en sus propiedades intrínsecas. Para ello, se han controlado los cambios en los valores de la capacidad de imbibición, porosidad abierta y total accesible al agua, coeficiente de absorción capilar y permeabilidad al vapor de agua, cuando las rocas "frescas" y envejecidas se someten a estos tratamientos.

\section{MATERIALES Y MÉTODOS}

Se han estudiado cinco facies graníticas naturales, dos de baja porosidad, correspondientes a facies no alteradas (M-1: granito gris de grano fino; $M-2$ : granito gris de grano grueso) y tres facies alteradas ( $M-3$ : granito ocre; M-4: granito rojo; $M-5$ : granito blanco); éstas son altamente porosas y con componentes muy reactivos (arcillas expandibles, ópalo, etc.). Su origen y sus propiedades mineralógicas (11), Tabla 1, químicas (12), Tabla 2, y petrofísicas (13), Tabla 3, ya han sido estudiadas. Todos estos materiales se han utilizado ampliamente en la construcción y reconstrucción de la Catedral y otros edificios de Ávila (España), que es una de las ciudades declaradas Patrimonio de la Humanidad por la UNESCO.

Se ensayaron los siguientes productos comerciales de tratamiento: RC70 (silicato de etilo) como consolidante,
A number of authors has determined hydric properties before and after application of consolidation and/or waterrepellency treatments (1-8) to evaluate their effectiveness. Martin et al. (2) designed a device that measures capillary and total water absorption, water sorption, desorption, and water vapour permeability, and features computerized monitoring and a thermostat to take readings over a wide range of temperatures.

Moreover, in the application of both silico-organic consolidants and polysiloxane water-repellents, natural stone transpiration is controlled by water vapour permeability. If permeability is extremely low, the flow of liquids is obstructed while gases and vapours are able to flow freely. The use of water-repellents pursues this effect, preventing liquids from penetrating while allowing the elimination of gas to preclude the increases in volume and concomitant generation of internal tension due to sudden rises in temperature (9).

Total water-accessible porosity of a material is the sum of "closed" porosity (not connected to the outside surface and scantly accessible to fluids) plus open porosity (externally connected and readily accessible to fluids). Open porosity, then, controls fluid transport through the stone at atmospheric temperature and pressure $(9,10)$.

The present study explores the effect of consolidation and/or water-repellency treatments on 5 samples of "fresh" granite facies, some previously aged in a climatic chamber under controlled conditions, through a minute analysis of changes in their intrinsic properties. To this end, changes in imbibition capacity, open and total water-accessible porosity, the capillary absorption coefficient and water vapour permeability were monitored when these treatments were applied to "fresh" and aged rocks.

\section{MATERIALS AND METHODS}

Five natural granite facies were studied, two with low porosity corresponding to unaltered facies (M-1: fine grain grey granite; M-2: coarse grain grey granite) and three highly porous with naturally altered facies (M-3: ochre granite; M-4: red granite; M-5: white granite) and very reactive components (swelling clays, opal and similar). Their origin and mineralogical (11) (Table 1), chemical (12) (Table 2) and petrophysical (13]) (Table 3) properties have been discussed in previous studies. All these materials have been widely used in the construction and reconstruction of Ávila Cathedral and other buildings in that Spanish city, which is a UNESCO World Heritage Site.

The following commercial products were tested: RC70 (ethyl silicate) as a consolidant, RC80 (a mixture of ethyl 
RC80 (una mezcla de silicato de etilo, una resina metílica y polisiloxano) como consolidante + hidrofugante y $\mathrm{H} 224$ (oligómero alquilpolisiloxánico) como hidrofugante, todos ellos suministrados por Rhône Poulenc.

Los tratamientos se llevaron a cabo como sigue:

a) Para el envejecimiento, se sometieron muestras cúbicas de $(5 \times 5 \times 5) \mathrm{cm}$ a 25 ciclos de hielo/deshielo junto silicate, methyl resin and polysiloxane) as consolidant and water-repellent and $\mathrm{H} 224$ (alkylpolysiloxane oligomer) as a water-repellent, all supplied by Rhône Poulenc.

The specimens were treated as follows:

a) For ageing, cubic specimens $(5 \times 5 \times 5) \mathrm{cm}$ were subjected to 25 freeze/thaw cycles (from -20 to 110

TABLA 1/ TABLE 1

Composición mineralógica de los materiales pétreos estudiados Mineralogical composition of the samples studied

\begin{tabular}{|ccccccccc|}
\hline $\begin{array}{c}\text { Muestra } \\
\text { (Sample) }\end{array}$ & $\mathrm{Q}$ & $\mathrm{F}$ & $\mathrm{M}$ & $\mathrm{C}$ & $\mathrm{S}$ & $\mathrm{K}$ & $\mathrm{O}$ & $\mathrm{OH}$ \\
\hline $\mathrm{M}-1$ & $\mathrm{xxx}$ & $\mathrm{xxx}$ & $\mathrm{xxx}$ & $\mathrm{x}$ & & & & \\
$\mathrm{M}-2$ & $\mathrm{xxx}$ & $\mathrm{xxx}$ & $\mathrm{xx}$ & $\mathrm{x}$ & & & & \\
$\mathrm{M}-3$ & $\mathrm{xxx}$ & $\mathrm{xx}$ & $\mathrm{x}$ & & $\mathrm{xx}$ & $\mathrm{x}$ & $\mathrm{x}$ & $\mathrm{t}$ \\
$\mathrm{M}-4$ & $\mathrm{xx}$ & & & & & $\mathrm{xx}$ & $\mathrm{xx}$ & $\mathrm{x}$ \\
$\mathrm{M}-5$ & $\mathrm{xx}$ & & & & & $\mathrm{xx}$ & $\mathrm{xx}$ & \\
\hline
\end{tabular}

$\mathrm{Q}=$ cuaryo (Quartz); $\mathrm{F}=$ feldespatos (feldspars); $\mathrm{M}=$ mica (mica); $\mathrm{C}=$ clorita (chlorite); $\mathrm{S}=\mathrm{esmectita}$ (smectite), $\mathrm{K}=$ kaolinita (kaolinite); $\mathrm{O}=\mathrm{o} p a l o$ (opal); $\mathrm{OH}=$ oxihidroxidos (oxyhydroxides) $\mathrm{xxx}=$ dominante $($ dominant $) ; \mathrm{xx}=\mathrm{abundante}$ (abundant); $\mathrm{x}=$ presente $($ present $) ; \mathrm{t}=$ trazas $($ traces $)$

TABLA 2/ TABLE 2

Composición química de los elementos mayores de las muestras estudiadas (\%) Chemical composition (major elements) in the samples studied (\%)

\begin{tabular}{|c|c|c|c|c|c|c|c|c|c|c|c|}
\hline $\begin{array}{c}\text { Muestra } \\
\text { (Sample) }\end{array}$ & $\mathrm{SiO}_{2}$ & $\mathrm{Al}_{2} \mathrm{O}_{3}$ & $\mathrm{Fe}_{2} \mathrm{O}_{3}$ & $\mathrm{CaO}$ & $\mathrm{MgO}$ & $\mathrm{Na}_{2} \mathrm{O}$ & $\mathrm{K}_{2} \mathrm{O}$ & $\mathrm{SO}_{3}$ & $\mathrm{TiO}_{2}$ & $\mathrm{MnO}$ & $\mathrm{P}_{2} \mathrm{O}_{5}$ \\
\hline $\mathrm{M}-1$ & 72.40 & 14.00 & 1.80 & 0.60 & 0.40 & 2.50 & 5.60 & 0.03 & 0.31 & 0.03 & 0.35 \\
\hline $\mathrm{M}-2$ & 66.60 & 16.10 & 3.70 & 3.40 & 1.20 & 3.10 & 3.70 & 0.09 & 0.53 & 0.06 & 0.26 \\
\hline $\mathrm{M}-3$ & 69.60 & 14.10 & 5.70 & 0.10 & 0.10 & 0.10 & 0.10 & 0.07 & 0.44 & 0.01 & 0.21 \\
\hline $\mathrm{M}-4$ & 73.20 & 13.80 & 3.10 & 0.04 & 0.10 & 0.20 & 0.20 & 0.04 & 0.41 & 0.01 & 0.15 \\
\hline $\mathrm{M}-5$ & 73.60 & 15.20 & 0.90 & 0.10 & 0.10 & 0.10 & 0.20 & 0.07 & 0.47 & 0.08 & 0.10 \\
\hline
\end{tabular}

TABLA 3/ TABLE 3

Propiedades hídricas de las muestras estudiadas Hydric properties of the samples studied

\begin{tabular}{|c|c|c|c|c|c|c|c|c|}
\hline $\begin{array}{c}\text { Muestra } \\
\text { (Sample) }\end{array}$ & DA* & DR* & PT** & PA** & CAA** & CI** & PVA*** & CAC**** \\
\hline M-1 & 2.60 & 2.63 & 1.12 & 1.02 & 91 & 0.47 & 2.26 & 7 \\
\hline M-2 & 2.66 & 2.68 & 0.65 & 0.62 & 95 & 0.29 & 2.44 & 5 \\
\hline M-3 & 1.77 & 2.47 & 28.40 & 21.02 & 74 & 12.78 & 47.55 & 140 \\
\hline M-4 & 1.98 & 2.48 & 20.13 & 13.49 & 67 & 7.92 & 25.59 & 80 \\
\hline M-5 & 1.87 & 2.37 & 21.04 & 12.62 & 60 & 8.25 & 35.62 & 70 \\
\hline
\end{tabular}

$\mathrm{DA}=$ densidad aparente (apparent density), DR=densidad real (real density), PT=porosidad total accesible al agua (total porosity accessible to water), $\mathrm{PA}=$ porosidad abierta (open porosity), CAA=coeficiente de absorción de agua (water absorption coefficient), CI=capacidad de imbibición (imbibition capacity), PVA=permeabilidad al vapor de agua (permeability to water vapour), $\mathrm{CAC}=$ coeficiente de absorción capilar (capillar absorption coefficient).

${ }^{*} \mathrm{~g} / \mathrm{cm}^{3} ;{ }^{* *} \%$; ${ }^{* * *} 10^{-6}\left(\mathrm{~kg} / \mathrm{m}^{2} \mathrm{~s}\right) ;{ }^{* * *} 10^{-5}\left(\mathrm{~g} / \mathrm{cm}^{2} \mathrm{~s}^{1 / 2}\right)$. 
frío/calor $\left(-20\right.$ a $\left.110^{\circ} \mathrm{C}\right)$ en una cámara climática, siguiendo las recomendaciones de Tiano y Pecchioni (14). Las muestras son sumergidas completamente en agua destilada durante 16 horas; después se enfrían a $-20^{\circ} \mathrm{C}$ y se mantiene esta temperatura durante 3 horas para, a continuación, elevar la temperatura a $110^{\circ} \mathrm{C}$, temperatura que se mantiene también durante 3 horas. Finalmente, se dejan las probetas 2 horas a temperatura ambiente y se reinicia el proceso.

b) Los tratamientos de consolidación se llevaron a cabo siguiendo las recomendaciones del NORMAL (15), aunque ligeramente modificadas, por inmersión de las muestras en el consolidante (en lugar de absorción capilar) y usando diferentes concentraciones (en lugar de una sola), como se describe a continuación, para favorecer la penetración del producto en el interior de la piedra. Los tratamientos se llevaron a cabo en cuatro fases consecutivas sin dejar tiempo entre ellas, inmersión de las muestras en white spirit (utilizado también como disolvente en los productos de tratamiento comerciales estudiados) durante 30 minutos, seguida por inmersiones en soluciones de concentraciones crecientes de los tratamientos en white spirit: (i) ocho horas con una solución del $5 \%$; (ii) veinticuatro horas con una solución del $40 \%$; (iii) cuarenta y ocho horas con una solución del $75 \%$.

c) El tratamiento de hidrofugación se aplicó sobre la superficie de la piedra con un pincel en dos aplicaciones sucesivas con un intervalo de 24 horas (16). En el caso de hidrofugación posterior al tratamiento de consolidación (RC70) o consolidación + hidrofugación (RC80), se dejan las probetas tras el primer tratamiento 20 días en condiciones ambientales ( $\mathrm{TH} \approx 20^{\circ} \mathrm{C}$ y humedad relativa $<60 \%$ ) y luego se les aplica el hidrofugante H224 tal y como se ha descrito anteriormente.

d) El análisis estadístico de los resultados se llevó a cabo con un diseño experimental completo de 3 factores de variación. Estos factores fueron:

(i) Tipo de muestra: con cinco niveles, referidos a los 5 tipos de muestra ( $M-1, M-2, M-3, M-4$ y $M-5)$.

(ii) Tratamiento: 6 niveles, correspondientes a la aplicación de hidrofugante (H224), dos consolidantes (RC70 y $\mathrm{RC} 80$, que contiene en su composición un agente hidrofugante), dos aplicaciones de consolidantes (RC70 y RC80) seguidas posteriormente del hidrofugante $\mathrm{H} 224$ y un control de las muestras no tratadas.

(iii) Envejecimiento artificial, con dos niveles, correspondiendo a las muestras envejecidas o no.

Las variables controladas para evaluar la eficacia de los tratamientos, fueron: capacidad de imbibición (17), porosidad abierta $(18,19)$ y total accesible al agua (18), coeficiente de absorción capilar (20) y permeabilidad al vapor de agua (21). Éstas se determinaron antes (piedras naturales o previamente envejecidas) y después de dar los tratamientos.

El estudio estadístico se realizó sobre 115 muestras (17 M-1, $17 \mathrm{M}-2,27 \mathrm{M}-3,27 \mathrm{M}-4$ y $27 \mathrm{M}-5)$. Dentro de las $\left.{ }^{\circ} \mathrm{C}\right)$ in a climatic chamber, as described by Tiano and Pecchioni (14). The samples were completely immersed in distilled water for 16 hours and chilled to and kept at $-20{ }^{\circ} \mathrm{C}$ for three hours, after which the temperature was raised to and maintained at $110^{\circ} \mathrm{C}$ for a further 3 hours. Subsequent to cooling at laboratory temperature for two hours, the process was repeated.

b) The consolidation treatments were conducted to the recommendations set out in NORMAL 20/85 (15), with certain modifications: the samples were immersed (instead of applying the substance by capillary absorption) in different (instead of only one) concentrations of the consolidant, as described below, to favour product penetration into the stone. The treatments were applied in four consecutive phases with no intermediate pauses: immersion in petroleum ether (also used as a solvent in the commercial products studied) for 30 minutes, followed by immersion in solutions with increasing concentrations of the treatments in white spirit: (i) eight hours in a 5\% solution; (ii) twenty four hours in a $40 \%$ solution; (iii) forty eight hours in a $75 \%$ solution.

c) Two successive coats of the water-repellency treatment were brushed on to the surface of the stone, the second 24 hours after the first (16). When the water-repellent was applied in addition to the consolidant (RC70) or the consolidant + water-repellent (RC80), after application of the first treatment the specimens were stored under laboratory conditions for 20 days $\left(T \approx 20^{\circ} \mathrm{C}\right.$ and relative humidity $<60 \%$ ) and then treated with the waterrepellent $\mathrm{H} 224$ as described above.

d) The experimental design used for the statistical analysis of the results addressed three factors of variation, namely:

(i) Type of sample: covering the 5 sample types (M-1, $M-2, M-3, M-4$ and $M-5$ ).

(ii) Treatment: covering the 6 possibilities - the waterrepellent ( $\mathrm{H} 224)$, the two consolidants ( $R C 70$ and $R C 80$, containing a water-repellent), each consolidant in conjunction with the $\mathrm{H} 224$ water-repellent and the untreated control.

(iii) Artificial ageing: covering aged and unaged specimens.

The variables monitored to evaluate treatment effectiveness were: imbibition capacity (17), open porosity $(18,19)$, total water-accessible porosity (18), capillar absorption coefficient (20) and water vapour permeability (21). The values of these parameters were determined for the natural or previously aged stone before and after applying the treatments.

The statistical study was conducted on 115 samples (17 M-1, $17 M-2,27 M-3,27 M-4$ and $27 M-5)$. Of the 17 
muestras $M-1$ y $M-2$, se tienen 5 muestras naturales y 12 previamente envejecidas antes de los tratamientos, mientras que en las muestras $M-3, M-4$ y $M-5$, se dispone de 15 naturales y 12 previamente envejecidas antes de los tratamientos.

\section{RESULTADOS}

Las muestras M-1 y M-2 ("frescas", granitos no alterados) muestran muy baja porosidad, incluso después de los envejecimientos, no observándose variaciones significativas en las propiedades estudiadas después de la aplicación de los tratamientos estudiados y, por ello, no se han incluido en los estudios estadísticos realizados.

El estudio estadístico llevado a cabo condujo a los siguientes resultados:

\section{Capacidad de imbibición}

En el análisis estadístico de los valores de esta variable, se detectó solamente la interacción (tipo de muestra) * (tipo de tratamiento) $(p=0,0018)$, Tabla 4. Tras su análisis, se detectaron diferencias altamente significativas $(P<0,01)$ entre la aplicación de cada uno de los tratamientos y las piedras no tratadas. También se detectaron diferencias, para todas las muestras, previamente envejecidas o no, entre la aplicación de los dos consolidantes (RC70 y RC80) y entre la del consolidante RC70 y el mismo con aplicación posterior del hidrofugante $\mathrm{H} 224$.

En el gráfico de la interacción, Figura 1, se comprueba que el tratamiento que más incide en la calidad del material (disminución de la capacidad de imbibición) es el uso de RC80, sólo o con aplicación posterior del hidrofugante $\mathrm{H} 224$, probablemente porque el RC80 contiene en su composición un agente hidrofugante activo. También, se observa que la muestra $M-3$, a pesar de disminuir su capacidad de
M-1 and M-2 samples, 5 were natural and 12 aged; and of the 27 in types $M-3, M-4$ and $M-5,15$ were natural and 12 aged.

\section{RESULTS}

Samples M-1 and M-2 ("fresh", unaltered granite) exhibited low porosity, even after ageing; as no significant variations were observed in the properties studied after applying the treatments, these samples were excluded from the statistical analyses.

The statistical study conducted led to the following results:

\section{Imbibition capacity}

The only interaction detected in the statistical analysis of the values for this variable was (type of sample) * (type of treatment) $(p=0.0018)$ (Table 4). The analysis of this interaction revealed highly significant differences (<0.01) between each of the treatments and the untreated stone. Differences were also detected for all samples, aged or otherwise, between the two consolidants (RC70 and RC80) and between consolidant RC70 alone and in conjunction with water-repellent $H 224$.

The interaction graph shown in Figure 1 confirms that the treatment with the greatest impact on material quality (decline in imbibition capacity) is RC80; this is observed irrespective of whether it is applied alone or together with a subsequent coat of $\mathrm{H224}$, probably because RC80 contains an active water-repellent. Despite the decrease in imbibition capacity after treatment, sample $M-3$

TABLA 4/ TABLE 4

Análisis ANOVA de tres vías para la capacidad de imbibición Three-Way ANOVA analysis for imbibition capacity

\begin{tabular}{|c|c|c|c|c|c|}
\hline $\begin{array}{l}\text { Fucnte de Variación } \\
\text { Source }\end{array}$ & $\begin{array}{l}\text { gl } \\
d f\end{array}$ & \begin{tabular}{|c|} 
Suma de \\
cuadrados \\
Sum of \\
Squares \\
\end{tabular} & $\begin{array}{c}\text { Cuadrados } \\
\text { Medios } \\
\text { Mean } \\
\text { Square } \\
\end{array}$ & $\begin{array}{l}\text { F-Valor } \\
F \text {-Value }\end{array}$ & $\begin{array}{l}\text { P-valor } \\
\text { P-Value }\end{array}$ \\
\hline MUESTR $A / S A M P L E$ & 4 & 1166,507 & 291,627 & 187,621 &, 0001 \\
\hline ENVEJECIMIFNTOAGISING & 1 & 1,917 & 1,917 & 1,233 & 27() 8 \\
\hline TRATAMIENTOITREATMENT & 5 & 237,614 & 47,523 & 30,574 &, 0001 \\
\hline $\begin{array}{l}\text { MUESTR A*ENVEJECIMIENTO } \\
\text { SAMPIE**AGEING }\end{array}$ & 4 & 5,520 & 1,380 & 888 & .4763 \\
\hline $\begin{array}{l}\text { MUESTRA*'RATAMIENTOO } \\
\text { SAMPLE*TREATMENT }\end{array}$ & 20 & 81,828 & 4,091 & 2,632 &, 0018 \\
\hline $\begin{array}{l}\text { ENVEJECIMIENTO*TRATAMIENTO } \\
\text { AGIEING }{ }^{*} T R E A T M E N T\end{array}$ & 5 & 14,033 & 2,807 & 1,806 &, 1241 \\
\hline $\begin{array}{l}\text { MUESTRA*ENVEIECIMIENTO } \\
\text { *TRATAMILNTO } \\
\text { SAMPIE*AGIEING*TREATMENT }\end{array}$ & 10 & 29,447 & 2,945 & 1,894 & ,0619, \\
\hline Rcsidual/Residual & 6.5 & 101,032 & 1,554 & & \\
\hline
\end{tabular}

Dependiente: \% CAPACIDAD DE IMBIBICIÓN.

Dependent: \% IMBIBITION CAPACITY. 


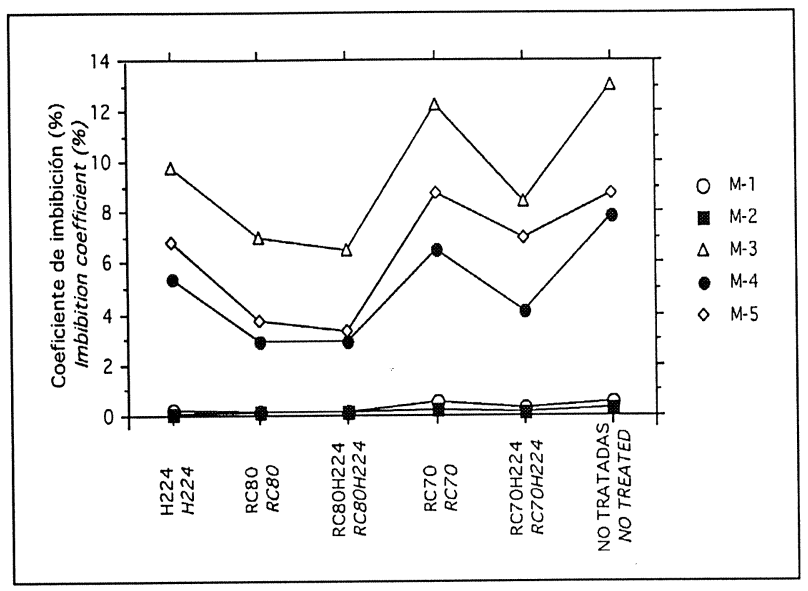

Figura 1.- Gráfico de la interacción (tipo de muestra * tipo de tratamiento) para la capacidad de imbibición.

Figure 1.- (Type of sample * type of treatment) interaction for imbibition capacity.

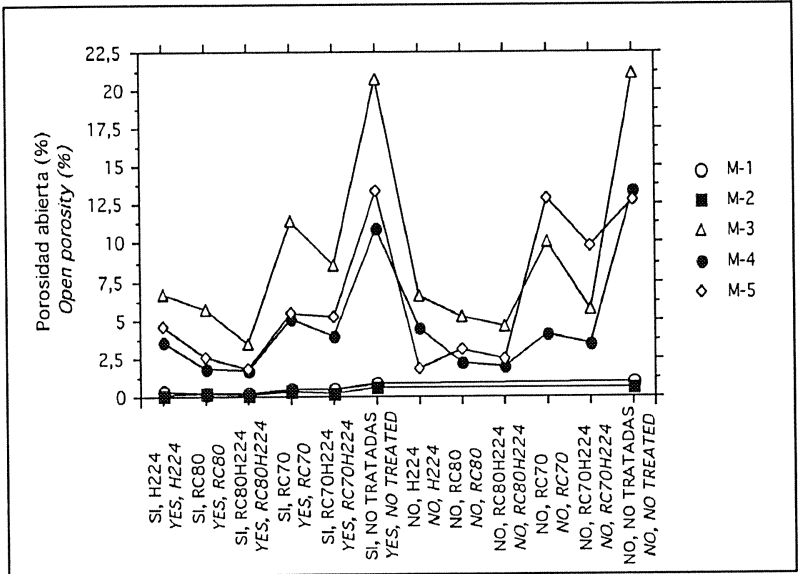

Figura 2.- Gráfico de la interacción (tipo de muestra * envejecimiento artificial * tipo de tratamiento) para la porosidad abierta.

Figure 2.- (Type of sample*artificial ageing * type of treatment) interaction for open porosity.

TABLA 5/ TABLE 5

Análisis ANOVA de tres vías para la Porosidad Abierta Three-Way ANOVA analysis for Open Porosity

\begin{tabular}{|c|c|c|c|c|c|}
\hline $\begin{array}{l}\text { Fuente de Variación } \\
\text { Source }\end{array}$ & $\begin{array}{l}\mathrm{gl} \\
d f\end{array}$ & \begin{tabular}{|c|} 
Suma de \\
cuadrados \\
Sum of \\
Squares \\
\end{tabular} & $\begin{array}{c}\text { Cuadrados } \\
\text { Medios } \\
\text { Mean } \\
\text { Square } \\
\end{array}$ & $\begin{array}{l}\text { I-Valor } \\
F-\text { Value }\end{array}$ & $\begin{array}{l}\text { P-valor } \\
\text { P-Value }\end{array}$ \\
\hline MUESTRA/SAMPLE & 4 & 1284,894 & 321,224 & 108,442 &, 0001 \\
\hline ENVEIIECIMIIENTO/AGEING & 1 & 8,594 & 8,594 & 2,901 &,$(0933$ \\
\hline TRATAMILNTOITRIEATMENT & 5 & 1056,399 & $211,28()$ & 71,326 &,$(0)(0) 1$ \\
\hline $\begin{array}{l}\text { MUESTRA*ENVEJECIMIENTO } \\
\text { SAMPLE*AGEING } \\
\end{array}$ & 4 & $15,4() 9$ & 3,852 & 1,300 &, 2792 \\
\hline $\begin{array}{l}\text { MUESTRA*TRATAMIENTO } \\
\text { SAMPLE*TREATMENT }\end{array}$ & 20 & 374,033 & 18,702 & 6,313 &, 0001 \\
\hline $\begin{array}{l}\text { ENVEIECIMIENTO*TR } A T A M I E N T O \\
\text { AGEING*TREATMENT } \\
\end{array}$ & 5 & 8,601 & 1,720 &, 581 & .7146 \\
\hline $\begin{array}{l}\text { MUESTRA*ENVILIECIMIENTO } \\
\text { *TRATAMIENTOO } \\
\text { SAMPLE*AGEING*TREATMENT }\end{array}$ & 10 & 84,071 & 8,407 & 2,838 &, 0055 \\
\hline Residual/Residual & 6.5 & 192,541 & 2,962 & & \\
\hline
\end{tabular}

Dependiente: \% POROSIDAD ABIERTA.

Dependent: \% OPEN POROSITY.

imbibición con los tratamientos, sigue absorbiendo más agua que el resto de las muestras, debido a su gran porosidad. Ambos hechos se observan en las demás propiedades estudiadas.

\section{Porosidad abierta}

En el análisis de la varianza, ANOVA, se detectó significación en la interacción triple (tipo de muestra) * (envejecimiento artificial) * (tipo de tratamiento), Tabla 5 , con $P=0.0055$. Después de su análisis, se obtuvieron similares resultados que para la capacidad de imbibición, Figura 2.

\section{Porosidad total accesible al agua}

En la Tabla 6, aparecen los resultados estadísticos obtenidos para esta variable. La interacción triple parece existir continued to absorb more water than any of the others, due to its high porosity. Both these patterns were also observed in the other properties studied.

\section{Open porosity}

ANOVA analysis detected a significant three-way interaction for this parameter (type of sample) * (artificial ageing) * (type of treatment) (Table 5) with $P=0.0055$. The results obtained after decomposition were similar to the results for imbibition capacity (Figure 2).

\section{Total water-accessible porosity}

Table 6 gives the statistical results obtained for this variable, for which a three-way interaction appears to 


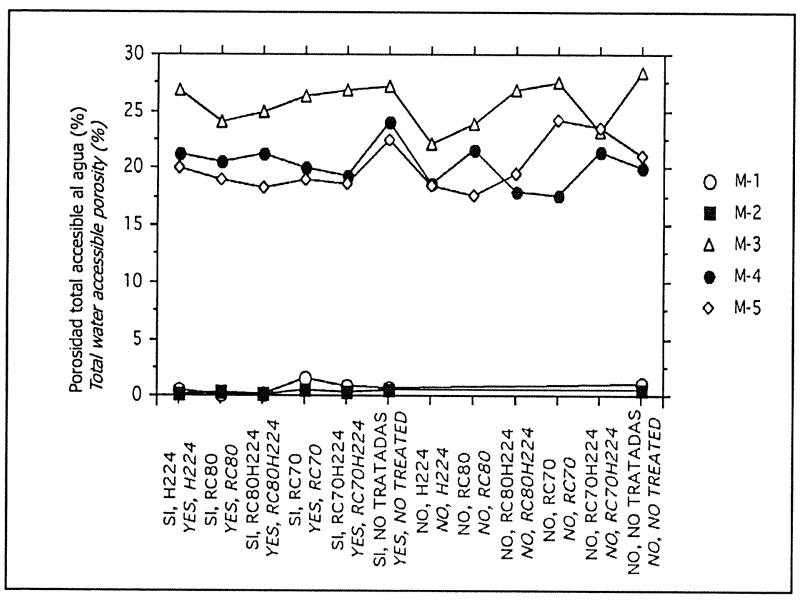

Figura 3.- Gráfico de la interacción (tipo de muestra * envejecimiento artificial * tipo de tratamiento) para la porosidad total accesible al agua.

Figure 3.- (Type of sample * artificial ageing * type of treatment) interaction for total water-accessible porosity.

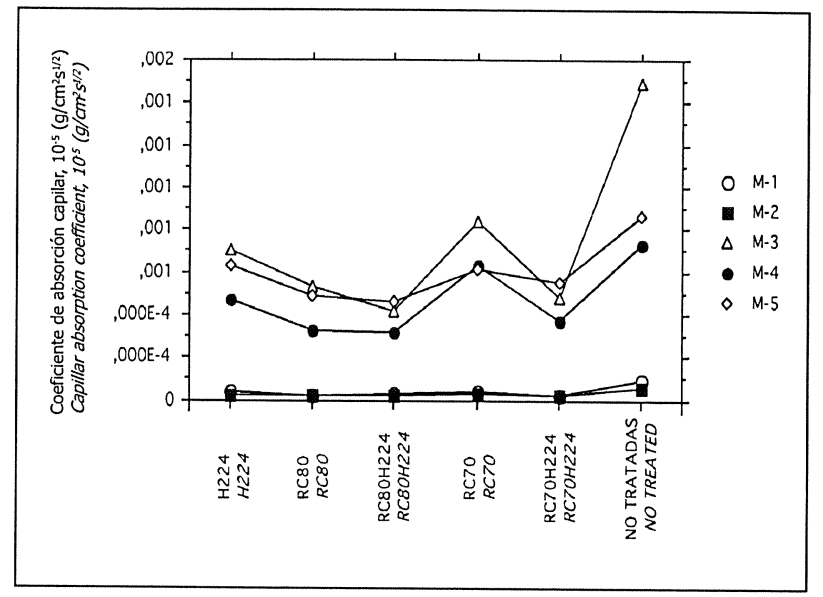

Figura 4.- Gráfico de la interacción (tipo de muestra * tipo de tratamiento) para el coeficiente de absorción capilar.

Figure 4. - (Type of sample * type of treatment) interaction for the capillary absorption coefficient.

TABLA 6/ TABLE 6

Análisis ANOVA de tres vías para la Porosidad Total Accesible al agua Three-Way ANOVA analysis for Total Water-Accessible Porosity

\begin{tabular}{|l|r|r|r|r|r|}
\hline $\begin{array}{l}\text { Fucnte de Variación } \\
\text { Source }\end{array}$ & $\begin{array}{c}\text { gl } \\
d f\end{array}$ & $\begin{array}{l}\text { Suma de } \\
\text { cuadrados } \\
\text { Sum of } \\
\text { Squares }\end{array}$ & $\begin{array}{c}\text { Cuadrados } \\
\text { Medios } \\
\text { Mean } \\
\text { Square }\end{array}$ & $\begin{array}{l}\text { F-Valor } \\
\text { F-Value }\end{array}$ & $\begin{array}{l}\text { P-valor } \\
\text { P-Value }\end{array}$ \\
\hline MUISSTRA/SAMPLE & 4 & 8821,560 & 2205,390 & 643,615 &, 0001 \\
\hline LNVLILCIMIENIOIAGEING & 1 & 1,643 & 1,643 &, 479 &, 4912 \\
\hline TRATAMIENTO!TREATMENT & 5 & 64,617 & 12,923 & 3,772 &, 0046 \\
\hline $\begin{array}{l}\text { MUESTRA*ENVEJECIMIENTO } \\
\text { SAMPLE*AGEING }\end{array}$ & 4 & 28,223 & 7,056 & 2,059 &, 0964 \\
\hline $\begin{array}{l}\text { MUESTRA*TRATAMIENTO } \\
\text { SAMPLE*TREATMENT }\end{array}$ & 20 & 68,942 & 3,447 & 1,006 &, 4679 \\
\hline $\begin{array}{l}\text { ENVEJECIMIENTO*TRATAMIENTO } \\
\text { AGEING*TREATMENT }\end{array}$ & 5 & 38,125 & 7,625 & 2,225 &, 0622 \\
\hline $\begin{array}{l}\text { MUESTRA*ENVEJECIMIENTO } \\
\text { *TRATAMIENTO } \\
\text { SAMPLE*AGEING*'TREATMENT }\end{array}$ & 10 & 86,954 & 8,695 & 2,538 &, 0120 \\
\hline ResidualiResidual & 6.5 & 222,727 & 3,427 & & \\
\hline
\end{tabular}

Dependiente: \% POROSIDAD TOTAL ACCESIBLE AL AGUA.

Dependent: \% TOTAL WATER-ACCESSIBLE POROSITY.

(tipo de muestra) * (envejecimiento artificial) * (tipo de tratamiento) $(P=0,012)$.

En el análisis de la interacción triple, después de los contrastes tras el ANOVA, se observaron diferencias $(P<0.01)$ entre las muestras envejecidas tratadas y las muestras no tratadas, excepto para el consolidante RC70, en el que las diferencias encontradas fueron del $5 \%$, y para el hidrofugante $\mathrm{H} 224$, en el que fueron casi despreciables. Además, se detectaron diferencias $(P<0,05)$ en las muestras no envejecidas con la aplicación del consolidante RC80 y la aplicación de RC80 y el hidrofugante H224, con respecto a las muestras no tratadas.

Estos resultados explican un efecto más notorio de los tratamientos sobre las muestras envejecidas, probablemente a exist (type of sample) * (artificial ageing) * (type of treatment $)(P=0.012)$.

The post hoc comparisons analyzing this three-way interaction revealed differences $(P<0.01)$ between the treated and untreated samples, except for consolidant RC70, where a 5\% difference was found, and for waterrepellent $\mathrm{H} 224$, for which differences were negligible. Moreover, differences $(P<0.05)$ were identified between the unaged samples treated with consolidant RC80 or RC80 plus the H224 water-repellent and the untreated samples.

These results explain the more conspicuous effect of the treatments on the aged samples, probably due to 
TABLA 7/TABLE 7

Análisis ANOVA de tres vías para el Coeficiente de Absorción Capilar Three-Way ANOVA analysis for Capillar Absorption Coefficient

\begin{tabular}{|l|r|r|r|r|r|}
\hline $\begin{array}{l}\text { Fucnte de Variación } \\
\text { Source }\end{array}$ & $\begin{array}{l}\mathrm{gl} \\
\mathrm{gl}\end{array}$ & $\begin{array}{l}\text { Suma de } \\
\text { cuadrados } \\
\text { Sum of } \\
\text { Squares }\end{array}$ & $\begin{array}{c}\text { Cuadrados } \\
\text { Mcdios } \\
\text { Mean } \\
\text { Square }\end{array}$ & $\begin{array}{l}\text { F-Valor } \\
\text { F-Value }\end{array}$ & $\begin{array}{r}\text { P-valor } \\
\text { P-Value }\end{array}$ \\
\hline MUESIRA/SAMPLE & 4 & $4,228 \mathrm{E}-6$ & $1,057 \mathrm{E}-6$ & 28,775 &, 0001 \\
\hline ENVEJEC.IMIENTO/AGEING & 1 & $1,937 \mathrm{E}-10$ & $1,937 \mathrm{E}-10$ &, 005 &, 9427 \\
\hline TRATAMIENTO/TREATMENT & 5 & $2,105 \mathrm{E}-6$ & $4,209 \mathrm{E}-7$ & 11,457 &, 0001 \\
\hline $\begin{array}{l}\text { MUESTRA*ENVEJECIMIENTO } \\
\text { SAMPLE*AGEING }\end{array}$ & 4 & $5,008 \mathrm{E}-8$ & $1,252 \mathrm{E}-8$ &, 341 &, 8476 \\
\hline $\begin{array}{l}\text { MUESTRA*TRATAMIENTO } \\
\text { SAMPLE*TREATMENT }\end{array}$ & 20 & $1,435 \mathrm{E}-6$ & $7,173 \mathrm{E}-8$ & 1,953 &, 0621 \\
\hline $\begin{array}{l}\text { ENVEJECIMIENTO*TRATAMIENTO } \\
\text { AGEING*TREATMENT }\end{array}$ & 5 & $1,459 \mathrm{E}-7$ & $2,918 \mathrm{E}-8$ &, 794 &, 5649 \\
\hline $\begin{array}{l}\text { MUESTRA*ENVEJECIMIENTOO } \\
\text { *'TRA'TAMIENTO } \\
\text { SAMPLE*AGEING*TREATMENT }\end{array}$ & 10 & $2,620 \mathrm{E}-7$ & $2,620 \mathrm{E}-8$ &, 713 &, 7038 \\
\hline Residual/Residual & 23 & $8,450 \mathrm{E}-7$ & $3,674 \mathrm{E}-8$ & & \\
\hline
\end{tabular}

Dependiente: COEFICIENTE DE ABSORCIÓN CAPILAR, $10^{-5}\left(\mathrm{~g} / \mathrm{cm}^{2} \mathrm{~s}^{1 / 2}\right)$.

Dependent: CAPILLAR ABSORPTION COEFFICIENT, $10^{-5}\left(\mathrm{~g} / \mathrm{cm}^{2} \mathrm{~s}^{1 / 2}\right)$.

causa de su mayor porosidad y el hecho de que ésta esté "mejor" conectada con la superficie externa de las piedras; es decir, que sea, sobretodo, porosidad abierta, Figura 3.

\section{Coeficiente de absorción capilar (CAC)}

Para esta variable, se ha detectado significación en la interacción (tipo de muestra) * (tipo de tratamiento) $(P=0,0621)$, Tabla 7 .

Se encontraron diferencias $(P<0,01)$, en los tests tras el ANOVA, entre los valores del CAC en las muestras tratadas (sea cual sea el tratamiento), si los comparamos con los de las muestras no tratadas. También, se detectaron diferencias $(P<0,05)$ entre ambos consolidantes (RC70 y RC80), y entre los valores obtenidos tras la aplicación del consolidante RC70, y la aplicación primero del RC70 y posteriormente el hidrofugante $\mathrm{H} 224$. Esta diferencia no se observó para el consolidante RC80, al indicar que no se había conseguido mejorar, cuando se utiliza posteriormente el hidrofugante (H224), Figura 4.

\section{Permeabilidad al vapor de agua}

En la Tabla 8, aparecen los resultados obtenidos en el estudio estadístico de esta variable, encontrándose diferencias entre las muestras $(P<0,01)$ y los tratamientos $(P>0,05)$. En la Figura 5, se ha preferido poner, a efectos de interpretación, el gráfico de la interacción doble (tipo de muestra) * (tipo de tratamiento), aunque en el modelo no exista significación en esta interacción, con el fin de ver los efectos de los factores principales citados (tipo de muestra y tipo de tratamiento).

El efecto de los diferentes tratamientos se ha estudiado siguiendo los test LSD y Bonferroni. Se detectaron diferencias $(P<0,01)$ en los contrastes LSD, cuando se aplica el consolidante RC80 y, posteriormente, la del their higher porosity and the fact that their pores are more externally "available"; i.e., that nearly all the porosity is open (Figure 3).

\section{Capillar absorption coefficient (CAC)}

Significant levels of (type of sample) * (type of treatment) interaction were detected for this variable $(P=0.0621)$ (Table 7).

In the post hoc comparisons, differences $(P<0.01)$ were found between the CAC values in the treated (regardless of type of treatment) and untreated samples. Differences $(P<0.05)$ were likewise identified between the two consolidants (RC70 and RC80) and between the values obtained for RC70 alone and in conjunction with waterrepellent $\mathrm{H} 224$. The fact that this difference was not observed for consolidant RC80 infers that no improvement was achieved with subsequent application of the waterrepellent (H224) in this case (Figure 4 ).

\section{Water vapour permeability}

Table 8 gives the results obtained in the statistical study for this variable, where differences were observed between the samples $(P<0.01)$ and the treatments $(P<0.05)$. The (type of sample)*(type of treatment) two-way interaction graph is depicted in Figure 5 to visualize the effects of the chief factors involved (type of sample and type of treatment), even though this interaction was not found to be significant in the model.

The effect of the various treatments was studied with the $\angle S D$ and Bonferroni tests. The $L S D$ test revealed differences $(P<0.01)$ between untreated samples and the specimens treated with consolidant RC80 in conjunction 
TABLA 8/ TABLE 8

Análisis ANOVA de tres vías para la Permeabilidad al Vapor de Agua Three-Way ANOVA analysis for Water Vapour Permeability

\begin{tabular}{|c|c|c|c|c|c|}
\hline $\begin{array}{l}\text { Fuente de Variación } \\
\text { Source }\end{array}$ & $\begin{array}{l}\mathrm{gl} \\
\mathrm{df}\end{array}$ & $\begin{array}{c}\text { Suma de } \\
\text { cuadrados } \\
\text { Sum of } \\
\text { Squares }\end{array}$ & $\begin{array}{l}\text { Cuadrados } \\
\text { Medios } \\
\text { Mean } \\
\text { Square }\end{array}$ & $\begin{array}{l}\text { F-Valor } \\
\text { F-Value }\end{array}$ & $\begin{array}{l}\text { P-valor } \\
\text { P-Value }\end{array}$ \\
\hline MULS'TRA/SAMI'LE & 4 & $8,639 \mathrm{E}-6$ & $2,160 \mathrm{E}-6$ & 56,342 &, 0009 \\
\hline ENVEJECIMIENTOIAGEING & 1 & $4,314 \mathrm{E}-9$ & $4,314 \mathrm{E}-9$ &, 113 &, 7541 \\
\hline TRAT AMIENTO/TREATMENT & 5 & $1,483 \mathrm{E}-6$ & $2,965 \mathrm{E}-7$ & 7,736 &, 0349 \\
\hline $\begin{array}{l}\text { MUUESTRA *ENVEJECIMIENTO } \\
\text { SAMPLE* }{ }^{*} \text { EGING }\end{array}$ & 4 & $1,333 \mathrm{E}-7$ & $3,332 \mathrm{E}-8$ &, 869 & $\overline{5524}$ \\
\hline $\begin{array}{l}\text { MUIESTRA*I'RATAMIIENIOO } \\
\text { SAMPLE*TREATMENT }\end{array}$ & 20 & $1,061 \mathrm{E}-6$ & $5,305 \mathrm{E}-8$ & 1,384 &, 4133 \\
\hline $\begin{array}{l}\text { ENVEJECIMIENTO*TRATAMIENTO } \\
\text { AGEING*TREATMENT }\end{array}$ & 5 & $1,410 \mathrm{E}-7$ & $2,821 \mathrm{E}-8$ &, 736 &, 6342 \\
\hline $\begin{array}{l}\text { MULSTRA*LNVIJIECIMIIENTO } \\
\text { *TRATAMIENTO } \\
\text { SAMPLE*AGEING*TREATMENT }\end{array}$ & 10 & $1,867 \mathrm{E}-7$ & $1,867 \mathrm{E}-8$ & 487 &, 8376 \\
\hline Residual/Residual & 4 & $1,533 \mathrm{E}-7$ & $3,833 \mathrm{E}-8$ & & \\
\hline
\end{tabular}

Dependiente: PERMEABILIDAD AL VAPOR DE AGUA, $10^{-6}\left(\mathrm{~kg} / \mathrm{m}^{2} \mathrm{~s}\right)$ Dependent: WATER VAPOUR PERMEABILITY, $10^{6}\left(\mathrm{~kg} / \mathrm{m}^{2} \mathrm{~s}\right)$.

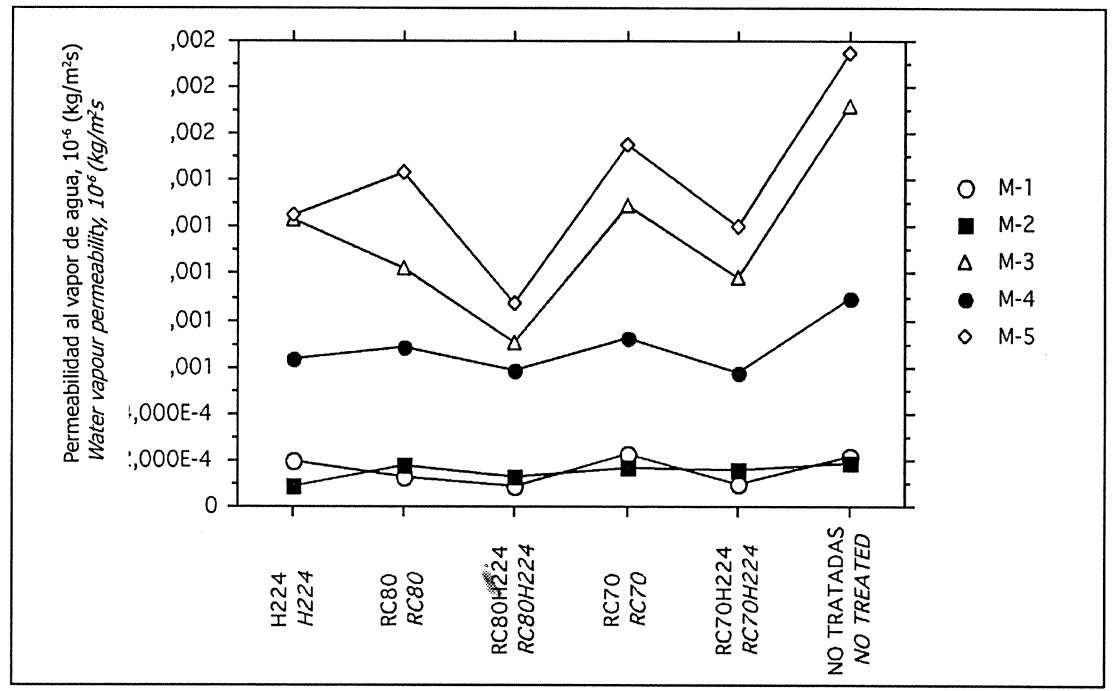

Figura 5.- Gráfico de la interacción (tipo de muestra * tipo de tratamiento) para la permeabilidad al vapor de agua.

Figure 5. - (Type of sample * type of treatment) interaction for water vapour permeability.

hidrofugante $\mathrm{H} 224$, si se compara con las muestras no tratadas. También, se encontraron diferencias $(P<0,05)$ entre el consolidante RC70 y el hidrofugante $\mathrm{H} 224$, cuando se compararon con las muestras no tratadas. El test Bonferroni no dio origen a ninguna diferencia al ser más restrictivo.

Estos resultados indican que la permeabilidad depende esencialmente del tipo de muestra sobre la que se aplica el tratamiento de superficie $y$, en menor medida, de la naturaleza específica del tratamiento dado.

\section{DISCUSIóN}

De los rèsultados obtenidos, se puede concluir que las mayores diferencias entre los distintos tratamientos realizados, se with a subsequent coating of water-repellent H224. Differences $(P<0.05)$ were also found between consolidant RC70-and water-repellent H224-treated samples and the untreated stone. The more restrictive Bonferroni test showed no differences.

These results indicate that permeability depends essentially on the type of sample to which the surface treatment is applied and, to a lesser extent, the specific nature of the treatment used.

\section{DISCUSSION}

According to the foregoing results, the parameters for which the differences between the various treatments were 
obtienen para la capacidad de imbibición y la porosidad abierta y, en menor extensión, para el coeficiente de absorción capilar. La capacidad de imbibición y el coeficiente de absorción capilar dependen de la estructura intrínseca del sólido, especialmente con la porosidad conectada con la superficie (porosidad abierta). Así, se podría recomendar el uso de la capacidad de imbibición, la porosidad abierta y el coeficiente de absorción capilar para evaluar los diferentes tratamientos.

En la determinación de la capacidad de imbibición (17) y porosidad abierta $(18,19)$ se absorbe agua a través de las 6 caras de las probetas cúbicas de las piedras; sin embargo, en la absorción capilar se absorbe solamente a través de una de las caras de los cubos (20), siendo la capacidad de absorción mucho más pequeña, lo que hace, en algunos casos, que las diferencias observadas en aquéllas se atenúen.

También se observó que en la porosidad total accesible al agua, las diferencias observadas en la porosidad abierta con los diferentes tratamientos desaparecían, porque la porosidad total accesible al agua tiene dos componentes, porosidad abierta y porosidad atrapada y esta última no se ve afectada por los tratamientos (22). Como consecuencia, a pesar de los cambios anteriores con los tratamientos (como se observa para las muestras $M-3, M-4$ y $M-5$, que tienen un alto contenido en arcillas y son altamente microporosas, con alta porosidad atrapada), resulta muy dependiente de la porosidad abierta. Así pues, la porosidad total accesible al agua no es recomendada como un parámetro para evaluar los tratamientos de conservación.

Además, se detectaron diferencias mayores con las muestras previamente envejecidas antes de tratarlas que con las que no lo habían sido. Este resultado no es tan inesperado, ya que los envejecimientos artificiales hacen crecer la relación entre la porosidad abierta y atrapada en las muestras estudiadas y la porosidad atrapada es más pequeña para las muestras envejecidas, porque se removiliza el material arcilloso original de la superficie de los poros, además de la formación de microfisuras intercristalinas e intracristalinas provocadas por los procesos de helacidad, a los que han sido sometidas las muestras. En este sentido, la red poral (directamente relacionada con la presencia de material arcilloso) cambia, aunque los poros que quedan en el material crezcan.

No se encontraron diferencias significativas en la permeabilidad al vapor de agua con los tratamientos estudiados; sin embargo, se encontraron diferencias entre las muestras estudiadas, indicando que los tratamientos no anulan la "transpiración" natural de las piedras.

\section{CONCLUSIONES}

La observación directa de los resultados y su análisis estadístico conduce a las siguientes conclusiones:

1) La determinación de la capacidad de imbibición, la porosidad abierta y el coeficiente de absorción capilar sirve para evaluar la eficacia de los tratamientos y, por tanto, largest were imbibition capacity and open porosity and to a lesser extent, the capillary absorption coefficient. Imbibition capacity and the capillary absorption coefficient depend on the intrinsic structure of the solid, particularly the externally available pores or open porosity. Hence, imbibition capacity, open porosity and the capillaryabsorption coefficient can be recommended as the parameters best suited to evaluating different treatments.

In the tests to determine imbibition capacity (17) and open porosity $(18,19)$, water is absorbed through all 6 sides of the cubic stone specimens. In CAC determination, however, water is absorbed through only one side of the cubes (20). The resulting substantial reduction in uptake attenuates the differences observed in some cases.

The differences in open porosity found between the various treatments disappeared in the findings for total wateraccessible porosity because the other component of this parameter, closed porosity, is not affected by the treatments (22). In other words, despite the changes described above after treatment (observed in samples M-3, M-4 and M-5, in which microporosity, clay content and closed porosity are high), total water-accessible porosity is extremely dependent on open porosity. Consequently, it cannot be recommended as a parameter for evaluating conservation treatments.

In another vein, greater differences were detected for aged than for unaged samples before and after treatment. This is not wholly unexpected, since artificial ageing increases the open / closed porosity ratio in the samples studied. Closed porosity is smaller in the aged samples because of the rearrangement of the original clay materials around the surface of the pores and the inter-and intracrystalline microcracking generated by the freezing processes to which the samples are subjected. In this regard, the porous network (directly related to the presence of clay materials) changes and the pores remaining in the material grow.

No significant differences were found in water vapour permeability for the treatments studied, although differences between the samples were identified, an indication that the treatments do not impact the natural "transpiration" of the stone.

\section{CONCLUSIONS}

The following conclusions can be drawn from direct observation of the results and their statistical analysis:

1) Imbibition capacity, open porosity and the capillary absorption coefficient are valid parameters for evaluating treatment effectiveness and therefore the quality of 
la calidad de los materiales antes y después de ser tratados. Así, en materiales graníticos porosos, como los aquí estudiados, el tratamiento combinado con silicatos orgánicos e hidrofugantes (polisiloxanos) mejoran sensiblemente sus propiedades como materiales de construcción.

2) La aplicación de consolidantes y posteriormente de hidrofugantes es más efectiva que sus acciones por separado, al menos con respecto a las propiedades hídricas ensayadas. La aplicación del hidrofugante (H224) disminuye el flujo de soluciones acuosas a través de la película formada sobre la superficie de las muestras, así baja la capacidad de imbibición, la porosidad abierta y el coeficiente de absorción capilar. Aunque en mucha menor extensión, la permeabilidad al vapor de agua también disminuye. En las propiedades hídricas determinadas, se demuestra que no es necesario aplicar el tratamiento $\mathrm{H} 224$, cuando ya se ha usado previamente el RC80, ya que no mejora su grado de hidrofugación.

3) La respuesta obtenida por diferentes materiales pétreos frente a un tratamiento depende de sus características intrínsecas, lo que hace muy difícil su predicción. Para ello, de antemano, se deberían hacer ensayos de cada material con cada tratamiento, bajo condiciones experimentales semejantes a las existentes en el monumento.

\section{AGRADECIMIENTOS}

Los autores agradecen la financiación de la Junta de Castilla y León (CSI08/03) y MEC (MAT2004-04498 y CGL2004-07066-CO2-02/BTE). Además, este trabajo se dedica a la memoria de la Dra. M. Angeles Vicente. the materials before and after treatment. Organic silicate treatments in conjunction with (polysiloxane) waterrepellents perceptibly improve the properties of porous granite such as studied here from the standpoint of their use as construction materials.

2) The application of consolidants followed by waterrepellency treatment is more effective than when either is used separately, in terms, at least, of the hydric properties studied. Applying water-repellent H224 decreases the flow of watery solutions through the film formed on the surface of the samples, thereby lowering imbibition capacity, open porosity and the capillary absorption coefficient. Water vapour permeability also declines, although to a much lesser extent. Waterrepellency is not enhanced by $\mathrm{H} 224$ after treatment with $R C 80$, rendering application of the water-repellency unnecessary in this case.

3) Since the response of stony materials to a given treatment depends on their intrinsic characteristics, results are difficult to predict. Each material should therefore be tested with each treatment under experimental conditions similar to those prevailing in the monument.

\section{ACKNOWLEDGEMENTS}

This research, funded by the Regional Government of Castile and Leon (CSI08/03) and MEC (MAT2004-04498 and CGL2004-07066-CO2-02/BTE). Is dedicated to the beloved memory of Dra. M. Angeles Vicente.

\section{BIBLIOGRAFÍA}

(1) F. García-Garmilla, I. Rodríguez-Maribona, M. Cano, M. Zalbide, J. A. Ibáñez-Gómez, E. Osa-Chans, S. Garín, «An analytical comparison of two comercial consolidating products applied to eocene sandstones from $16^{\text {th }}$ and $19^{\text {th }}$ century monuments in San Sebastián, northern Spain», Mater. Construcc., Vol. 52, no 266 (2002), pp. 5-18.

(2) J. Martín, F. J. Navas, R. Alcántara, «An instrumental system for continuous measuring of the sorption properties in porous materials». Proceedings of $5^{\text {th }}$ International Symposium on the Conservation of Monuments in the Mediterranean Basin «Protection and Conservation of the Cultural Heritage of the Mediterranean Cities», Sevilla, España (2002), pp. 327-330.

(3) R. Villegas-Sánchez, J. Espinosa-Gaitán, «Evaluation of treatment products for the front door of the church of Santiago, Guadix», Mater. Construcc., Vol. 51, no 261 (2001), pp. 21-32.

(4) I. Rodríguez-Maribona, M. Zalbide, F. García-Garmilla, J. A. Ibáñez, S. Garín, «Conservation study of the stone material used in the Culture House of Almirante Oquendo, in San Sebastián», Mater. Construcc., Vol. 49, no 255 (1999), pp. 19-30.

(5) E. Berardi, A. M. Mecchi, A. Calia, M. Lettieri, «Water-repellent treatments on some calcareous Apulian stones of differing porosity». Proceedings of $5^{\text {th }}$ International Symposium on the Conservation of Monuments in the Mediterranean Basin «Protection and Conservation of the Cultural Heritage of the Mediterranean Cities», Sevilla, España (2002), pp. 401-406.

(6) J. Espinosa-Gaitán, E. Ontiveros-Ortega, R. Villegas-Sánchez, M. Alcalde-Moreno, «Evaluation of treatments for the stone of the Córdoba Door of Carmona (Seville, España)». Proceedings of $5^{\text {th }}$ International Symposium on the Conservation of Monuments in the Mediterranean Basin «Protection and Conservation of the Cultural Heritage of the Mediterranean Cities», Sevilla, España (2002), pp.
431-436.

(7) R. Quaresima, G. Scoccia, R. Volpe, G. Toscani, «Behaviour of different treated and untreated stones exposed to salt crystallization test». Proceedings of $5^{\text {th }}$ International Symposium on the Conservation of Monuments in the Mediterranean Basin «Protection and Conservation of the Cultural Heritage of the Mediterranean Cities», Sevilla, España (2002), pp. 455-460.

(8) R. M. Esbert, J. Alonso, F. J. Alonso, J. Ordaz, «Suggested properties for evaluating the behavour of a waterproofed limestone under aggressive environmental conditions». Proceedings of International Colloquium on Methods of evaluating products for the conservation of porous building materials in monuments, Roma, Italia (1995), pp. 331-339.

(9) C. Brandes, E. Stadlbauer, «Capillary water absorption of painted stone». Proceedings of the 7th Int. Cong. on the Deterioration and Conservation of Stone, Lisboa, Portugal, Vol. 2 (1992), pp. 591-600.

(10) B. Meng, «Moisture-transport-relevant characterization of pore structure». Proceedings of the 7 th Int. Cong. on the Deterioration and Conservation of Stone, Lisboa, Portugal, Vol. 1 (1992), pp. 387-396. 
(11) J. García-Talegón, E. Molina, M. A. Vicente, «Weathering processes in granites». Proceedings of the 7th Cong. EUROCLAY, Dresden, Alemania, Vol. 2 (1991), pp. 405-409.

(12) J. García-Talegón, A. C. Iñigo, E. Molina, J. L. Pérez-Rodríguez, M. Vargas, M. A. Vicente, «Granites employed in Avila (Spain): I.- Chemical composition of the different types», Mater. Construcc., Vol. 44, no 233 (1994), pp. 23-28.

(13) A. C. Íñigo, J. García-Talegón, E. Molina, J. L. Pérez-Rodríguez, M. Vargas, M. A. Vicente, «Granites employed in Avila (Spain): II.- Petrophysical characteristics», Mater. Construcc., Vol. 44, no 233 (1994), pp. 28-37.

(14) P. Tiano, E. Pecchioni, «Invecchiamento artificiale di materiali lapidei». Proceedings of the Giornata di Studio «Camera climatiche od ambientali nella ricerca applicata», Florencia, Italia (1990), pp. 37-42.

(15) NORMAL 20/85, «Interventi conservativi: Progettazione esecuzione e valutazione preventiva», CNR-ICR, Roma, 1986a.

(16) R. M. Esbert, C. Grossi, J. Ordaz, F. J. Alonso, «La conservación de la piedra de la Casa Milá ('La Pedrera' de Gaudí, Barcelona): Pruebas preliminares», Boletín Geológico y Minero, Vol. 102-103 (1991), pp. 446-454.

(17) NORMAL 7/81, «Assorbimento d'acqua per immersione totale, capacita' di imbibizione», CNR-ICR, Roma, 1981.

(18) NF B10-503, «Mesures de la Porosité, de la Masse Volumique Réelle et de la Masse Volumique Apparente», $1973 a$.

(19) NF B10-504, «Mesure du Coefficient de Absortion d'Eau», 1973b.

(20) NORMAL 11/85, «Assorbimento d'acqua per capillaritá, coefficiente di assorbimento capillare», CNR-ICR, Roma, 1986b.

(21) RILEM. 25-PEM, «Coefficient de conductivité de vapeur d'eau», Mater. Struct., Vol. 13, nº 75 (1980), pp. 196-198.

(22) A. C. Íñigo, S. Vicente-Tavera, V. Rives, M. A. Vicente, «Porosidad libre en granitos alterados: Comentarios a las normas», Mater. Construcc., Vol. 45, no 238 (1995), pp. 47-53. 